\title{
Translation invariance and scale invariance of approximations of fuzzy numbers
}

\author{
Adrian Ban $^{1}$ Lucian Coroianu ${ }^{2}$ \\ ${ }^{1,2}$ Department of Mathematics and Informatics, University of Oradea, \\ Universităţii 1, 410087 Oradea, Romania \\ e-mails: aiban@uoradea.ro, lcoroianu@uoradea.ro
}

\begin{abstract}
We give some sufficient conditions such that a crisp, interval, triangular, trapezoidal, parametric approximation of a fuzzy number, with or without additional requirements, to have the properties of invariance to translations or scale invariance.
\end{abstract}

Keywords: Fuzzy number, Approximation, Scale invariance, Translation invariance.

\section{Introduction}

Lists of criteria which a crisp approximation (or defuzzification) and a trapezoidal approximation operator should or just possess were proposed in [13] and [10]. Scale invariance and translation invariance were included in these lists and then studied for recent introduced approximations of fuzzy numbers (e.g. [1] - [4], [18], [19]). The proofs are rather sophisticated when the approximation operators are given on cases. Our aim is to give some sufficient conditions, valid in a general framework, which ensure the scale and translation invariance.

\section{Preliminaries}

A fuzzy number $A$ is a fuzzy subset of the real line $\mathbb{R}$ with the membership function $\mu_{A}$ which is normal, fuzzy convex, upper semicontinuous and supp $A$ is bounded, where

$$
\operatorname{supp} A=\operatorname{cl}\left\{x \in \mathbb{R}: \mu_{A}(x)>0\right\}
$$

and $c l$ is the closure operator. A space of all fuzzy numbers will be denoted by $F(\mathbb{R})$.

Every $\alpha$-cut, $\alpha \in] 0,1]$, of $A \in F(\mathbb{R})$ is a closed interval $A_{\alpha}=\left[A_{L}(\alpha), A_{U}(\alpha)\right]$, where

$$
\begin{aligned}
& A_{L}(\alpha)=\inf \left\{x \in \mathbb{R}: \mu_{A}(x) \geq \alpha\right\}, \\
& A_{U}(\alpha)=\sup \left\{x \in \mathbb{R}: \mu_{A}(x) \geq \alpha\right\} .
\end{aligned}
$$

We denote

$$
A_{0}=\left[A_{L}(0), A_{U}(0)\right]=\operatorname{supp} A .
$$

For $A \in F(\mathbb{R})$,

$$
A_{\alpha}=\left[A_{L}(\alpha), A_{U}(\alpha)\right], \alpha \in[0,1]
$$

and $z, \lambda \in \mathbb{R}$ we consider the translation $A+z$ and the scalar multiplication $\lambda \cdot A$ by

$$
(A+z)_{\alpha}=A_{\alpha}+z=\left[A_{L}(\alpha)+z, A_{U}(\alpha)+z\right]
$$

and

$$
(\lambda \cdot A)_{\alpha}=\lambda A_{\alpha}=\left\{\begin{array}{l}
{\left[\lambda A_{L}(\alpha), \lambda A_{U}(\alpha)\right], \text { if } \lambda \geq 0} \\
{\left[\lambda A_{U}(\alpha), \lambda A_{L}(\alpha)\right], \text { if } \lambda<0}
\end{array}\right.
$$

Various characteristics of fuzzy numbers are useful in applications. Some of the most important are the expected interval $E I(A)$, expected value $E V(A)$, value $\operatorname{Val}(A)$, ambiguity $A m b(A)$ and width $w(A)$ of a fuzzy number $A$. They are given by (see [6], [8], [12])

$$
\begin{aligned}
E I(A) & =\left[\int_{0}^{1} A_{L}(\alpha) d \alpha, \int_{0}^{1} A_{U}(\alpha) d \alpha\right] \\
E V(A) & =\frac{1}{2}\left(\int_{0}^{1} A_{L}(\alpha) d \alpha+\int_{0}^{1} A_{U}(\alpha) d \alpha\right), \\
\operatorname{Val}(A) & =\int_{0}^{1} \alpha A_{U}(\alpha) d \alpha+\int_{0}^{1} \alpha A_{L}(\alpha) d \alpha \\
\operatorname{Amb}(A) & =\int_{0}^{1} \alpha A_{U}(\alpha) d \alpha-\int_{0}^{1} \alpha A_{L}(\alpha) d \alpha
\end{aligned}
$$

and

$$
w(A)=\int_{0}^{1} A_{U}(\alpha) d \alpha-\int_{0}^{1} A_{L}(\alpha) d \alpha .
$$

The weighted $L_{2}$-distance on $F(\mathbb{R})$ is defined as (see e.g. [18])

$$
\begin{aligned}
d_{\lambda}^{2}(A, B) & =\int_{0}^{1}\left(A_{L}(\alpha)-B_{L}(\alpha)\right)^{2} \lambda_{L}(\alpha) d \alpha \\
& +\int_{0}^{1}\left(A_{U}(\alpha)-B_{U}(\alpha)\right)^{2} \lambda_{U}(\alpha) d \alpha
\end{aligned}
$$

where $\lambda_{L}, \lambda_{U}$ are non-negative functions on $[0,1]$, called weighted functions, such that

$$
\int_{0}^{1} \lambda_{L}(\alpha) d \alpha>0
$$

and

$$
\int_{0}^{1} \lambda_{U}(\alpha) d \alpha>0
$$


If $\lambda_{L}(\alpha)=\lambda_{U}(\alpha)=1$, for every $\alpha \in[0,1]$, we obtain the $L_{2}$-distance on $F(\mathbb{R})$,

$$
\begin{aligned}
d^{2}(A, B) & =\int_{0}^{1}\left(A_{L}(\alpha)-B_{L}(\alpha)\right)^{2} d \alpha \\
& +\int_{0}^{1}\left(A_{U}(\alpha)-B_{U}(\alpha)\right)^{2} d \alpha .
\end{aligned}
$$

The $\delta_{p, q}$ distance, indexed by parameters $1 \leq p \leq$ $\infty, 0 \leq q \leq 1$, is given as follows (see [9])

$$
\begin{aligned}
\left(\delta_{p, q}(A, B)\right)^{p} & =(1-q) \int_{0}^{1}\left|A_{L}(\alpha)-B_{L}(\alpha)\right|^{p} d \alpha \\
& +q \int_{0}^{1}\left|A_{U}(\alpha)-B_{U}(\alpha)\right|^{p} d \alpha,
\end{aligned}
$$

for $1 \leq p<\infty$, and

$$
\begin{aligned}
\delta_{\infty, q}(A, B) & =(1-q) \sup _{0<\alpha \leq 1}\left|A_{L}(\alpha)-B_{L}(\alpha)\right| \\
& +q \sup _{0<\alpha \leq 1}\left|A_{U}(\alpha)-B_{U}(\alpha)\right| .
\end{aligned}
$$

The supremum distance $d_{\infty}$ and $L_{p, \infty}$-distances $d_{p}, 1 \leq p<\infty$, on $F(\mathbb{R})$, involve the Hausdorff metric between the $\alpha$-cuts of fuzzy numbers. They are defined by (see [7], p.52)

$$
\begin{aligned}
d_{\infty}(A, B) & =\sup _{0 \leq \alpha \leq 1} d_{H}\left(A_{\alpha}, B_{\alpha}\right) \\
d_{p}(A, B) & =\left(\int_{0}^{1}\left(d_{H}\left(A_{\alpha}, B_{\alpha}\right)\right)^{p} d \alpha\right)^{1 / p}
\end{aligned}
$$

where

$$
\begin{aligned}
& d_{H}\left(A_{\alpha}, B_{\alpha}\right) \\
& =\max \left\{\left|A_{L}(\alpha)-B_{L}(\alpha)\right|,\left|A_{U}(\alpha)-B_{U}(\alpha)\right|\right\} .
\end{aligned}
$$

A class of distances between fuzzy numbers was introduced in [5] by

$$
\widetilde{D}_{f, \varphi}(A, B)=\left(\int_{0}^{1} \widetilde{D}_{f}^{2}\left(A_{\alpha}, B_{\alpha}\right) d \varphi(\alpha)\right)^{1 / 2},
$$

where

$$
\begin{aligned}
& \widetilde{D}_{f}^{2}([a, b],[c, d]) \\
& =\int_{0}^{1}(t|a-c|+(1-t)|b-d|)^{2} d f(t),
\end{aligned}
$$

$f$ is a normalized weight measure on $[0,1]$ and usually the function $\varphi$ satisfies the conditions

$$
\begin{gathered}
\varphi(\alpha) \geq 0, \forall \alpha \in[0,1], \\
\alpha_{1} \leq \alpha_{2} \Rightarrow \varphi\left(\alpha_{1}\right) \leq \varphi\left(\alpha_{2}\right), \\
\int_{0}^{1} \varphi(\alpha) d \alpha=1 .
\end{gathered}
$$

On the basis of expressing the above metric $\widetilde{D}_{f}$ in terms of the mid and spread of intervals, the distance $D_{\psi, \theta}^{*}$ between fuzzy numbers was introduced in [16] as follows:

$$
D_{\psi, \theta}^{*}(A, B)=\left(\int_{0}^{1}\left(D_{\theta}^{*}\left(A_{\alpha}, B_{\alpha}\right)\right)^{2} d \psi(\alpha)\right)^{1 / 2}
$$

where $\theta \in(0,1], \psi$ is a weight probability measure on $[0,1]$,

$$
\begin{gathered}
\left(D_{\theta}^{*}([a, b],[c, d])\right)^{2}=(\operatorname{mid}[a, b]-\operatorname{mid}[c, d])^{2} \\
+\theta(\operatorname{spr}[a, b]-\operatorname{spr}[c, d])^{2}, \\
\operatorname{mid}\left[a_{1}, a_{2}\right]=\frac{a_{1}+a_{2}}{2}
\end{gathered}
$$

and

$$
\operatorname{spr}\left[a_{1}, a_{2}\right]=\frac{a_{2}-a_{1}}{2} .
$$

We recall, a distance $D$ on $F(\mathbb{R})$ is translation invariant if

$$
D(A+z, B+z)=D(A, B),
$$

for every $A, B \in F(\mathbb{R}), z \in \mathbb{R}$ and scale invariant if

$$
D(\lambda \cdot A, \lambda \cdot B)=|\lambda| D(A, B),
$$

for every $A, B \in F(\mathbb{R}), \lambda \in \mathbb{R} \backslash\{0\}$.

\section{Invariance to translations}

The translation invariance of an approximation operator $O$ on $F(\mathbb{R})$, that is

$$
O(A+z)=O(A)+z,
$$

for every $A \in F(\mathbb{R})$ and $z \in \mathbb{R}$, is considered a natural requirement. The following result gives sufficient conditions for the translation invariance of an operator which associates to a fuzzy number the nearest fuzzy number in a subset of fuzzy numbers, under additional conditions.

Theorem 1 Let $D$ be a translation invariant distance on $F(\mathbb{R})$ and $P_{k}, k \in\{1, \ldots, n\}$ is a real parameter or interval associated with fuzzy numbers such that

$$
P_{k}(A+z)=P_{k}(A),
$$

for every $A \in F(\mathbb{R})$ and $z \in \mathbb{R}$ or

$$
P_{k}(A+z)=P_{k}(A)+z,
$$

for every $A \in F(\mathbb{R})$ and $z \in \mathbb{R}$. If $\Omega \subset F(\mathbb{R})$ satisfies $z+\Omega=\Omega, \forall z \in \mathbb{R}$ and $\omega(A) \in \Omega$ is the nearest fuzzy number to a given $A \in F(\mathbb{R})$ (with respect to D) which preserves $P_{k}, k \in\{1, \ldots, n\}$, that is

$$
P_{k}(\omega(A))=P_{k}(A), \forall k \in\{1, \ldots, n\}
$$

then $\omega(A)+z \in \Omega$ is the nearest fuzzy number to $A+z$ (with respect to $D$ ) which preserves $P_{k}, k \in$ $\{1, \ldots, n\}$, that is

$$
P_{k}(\omega(A+z))=P_{k}(A+z), \forall k \in\{1, \ldots, n\} .
$$


Proof. We have

$$
D(A, \omega(A)) \leq D(A, B),
$$

for every $B \in \Omega$ such that $P_{k}(A)=P_{k}(B), \forall k \in$ $\{1, \ldots, n\}$ and

$$
P_{k}(A)=P_{k}(\omega(A)), \forall k \in\{1, \ldots, n\} .
$$

Let $B^{\prime} \in \Omega$ and let $z \in \mathbb{R}$ such that $P_{k}(A+z)=$ $P_{k}\left(B^{\prime}\right), \forall k \in\{1, \ldots, n\}$. Let $B \in \Omega$ such that $B^{\prime}=$ $B+z$. We get $P_{k}(A+z)=P_{k}(B+z)$ and taking into account $(9),(10)$ and the translation invariance of $D$ we get

$$
\begin{gathered}
D(A+z, \omega(A)+z)=D(A, \omega(A)) \leq D(A, B) \\
=D(A+z, B+z)=D\left(A+z, B^{\prime}\right) .
\end{gathered}
$$

In addition (taking into account (9) and (10) too),

$$
P_{k}(A+z)=P_{k}(\omega(A)+z)
$$

therefore $\omega(A)+z \in \Omega$ is the nearest element to $A+$ $z$ (with respect to $d$ ) which preserves the parameters $P_{k}, k \in\{1, \ldots, n\}$. In other words,

$$
\omega(A+z)=\omega(A)+z
$$

that is the operator $\omega$ is translation invariant.

Corollary 2 Let $P_{k}: F(\mathbb{R}) \rightarrow \mathbb{R}, k \in\{1, \ldots, n\}$ defined by

$$
\begin{aligned}
P_{k}(A) & =a_{k} \int_{0}^{1} A_{L}(\alpha) d \alpha+b_{k} \int_{0}^{1} A_{U}(\alpha) d \alpha \\
& +g_{k} \int_{0}^{1} \alpha A_{L}(\alpha) d \alpha+h_{k} \int_{0}^{1} \alpha A_{U}(\alpha) d \alpha,
\end{aligned}
$$

where $a_{k}, b_{k}, g_{k}, h_{k} \in \mathbb{R}, \Omega \subset F(\mathbb{R})$ with $z+\Omega=$ $\Omega, \forall z \in \mathbb{R}$ and $\omega: F(\mathbb{R}) \rightarrow \Omega$ such that $\omega(A)$ is the nearest fuzzy number to a given $A$ with respect to a translation invariant distance on $F(\mathbb{R})$, which, in addition, preserves the parameters $P_{k}, k \in$ $\{1, \ldots, n\}$. If

$$
2\left(a_{k}+b_{k}\right)+g_{k}+h_{k}=0
$$

or

$$
2\left(a_{k}+b_{k}\right)+g_{k}+h_{k}=2,
$$

for every $k \in\{1, \ldots, n\}$, then

$$
\omega(A+z)=\omega(A)+z,
$$

for every $z \in \mathbb{R}$.

Proof. In this particular case (9) is equivalent with (11) and (10) is equivalent with (12).

We denote

$$
\operatorname{Int}(\mathbb{R})=\{[a, b]: a, b \in \mathbb{R}, a<b\}
$$

Corollary 3 Let $P_{k}: F(\mathbb{R}) \rightarrow \operatorname{Int}(\mathbb{R}), k \in$ $\{1, \ldots, n\}$ defined by

$$
P_{k}(A)=\left[P_{k}^{\prime}(A), P_{k}^{\prime \prime}(A)\right],
$$

where

$$
\begin{aligned}
P_{k}^{\prime}(A) & =a_{k}^{\prime} \int_{0}^{1} A_{L}(\alpha) d \alpha+b_{k}^{\prime} \int_{0}^{1} A_{U}(\alpha) d \alpha \\
& +g_{k}^{\prime} \int_{0}^{1} \alpha A_{L}(\alpha) d \alpha+h_{k}^{\prime} \int_{0}^{1} \alpha A_{U}(\alpha) d \alpha, \\
P_{k}^{\prime \prime}(A) & =a_{k}^{\prime \prime} \int_{0}^{1} A_{L}(\alpha) d \alpha+b_{k}^{\prime \prime} \int_{0}^{1} A_{U}(\alpha) d \alpha \\
& +g_{k}^{\prime \prime} \int_{0}^{1} \alpha A_{L}(\alpha) d \alpha+h_{k}^{\prime \prime} \int_{0}^{1} \alpha A_{U}(\alpha) d \alpha,
\end{aligned}
$$

$a_{k}^{\prime}, b_{k}^{\prime}, g_{k}^{\prime}, h_{k}^{\prime}, a_{k}^{\prime \prime}, b_{k}^{\prime \prime}, g_{k}^{\prime \prime}, h_{k}^{\prime \prime} \in \mathbb{R}, \Omega \subset F(\mathbb{R})$ such that $z+\Omega=\Omega$ and $\omega: F(\mathbb{R}) \rightarrow \Omega$ such that $\omega(A)$ is the nearest fuzzy number to $A$ with respect to a translation invariant distance on $F(\mathbb{R})$ which, in addition, preserves $P_{k}, k \in\{1, \ldots, n\}$. If

$$
\begin{aligned}
& 2\left(a_{k}^{\prime}+b_{k}^{\prime}\right)+g_{k}^{\prime}+h_{k}^{\prime}=0 \\
& 2\left(a_{k}^{\prime \prime}+b_{k}^{\prime \prime}\right)+g_{k}^{\prime \prime}+h_{k}^{\prime \prime}=0
\end{aligned}
$$

or

$$
\begin{aligned}
& 2\left(a_{k}^{\prime}+b_{k}^{\prime}\right)+g_{k}^{\prime}+h_{k}^{\prime}=2, \\
& 2\left(a_{k}^{\prime \prime}+b_{k}^{\prime \prime}\right)+g_{k}^{\prime \prime}+h_{k}^{\prime \prime}=2,
\end{aligned}
$$

for every $k \in\{1, \ldots, n\}$, then

$$
\omega(A+z)=\omega(A)+z,
$$

for every $z \in \mathbb{R}$.

Proof. In this particular case (9) is equivalent with (13)-(14) and (10) is equivalent with (15)-(16).

\section{Scale invariance}

It is well-known that an operator $O$ on $F(\mathbb{R})$ is scale invariant if

$$
O(\lambda \cdot A)=\lambda \cdot O(A),
$$

for every $A \in F(\mathbb{R})$ and $\lambda \in \mathbb{R}$. The scale invariance is a basic requirement for any approximation operator on $F(\mathbb{R})$ (see [10], [13]). The result corresponding to Theorem 1 is the following.

Theorem 4 Let $D$ be a scale invariant distance on $F(\mathbb{R})$ and $P_{k}, k \in\{1, \ldots, n\}$ real parameters or intervals associated to fuzzy numbers such that

$$
P_{k}(\lambda \cdot A)=\lambda P_{k}(A),
$$

for every $A \in F(\mathbb{R})$ and $\lambda \in \mathbb{R}$ or

$$
P_{k}(\lambda \cdot A)=|\lambda| P_{k}(A),
$$


for every $A \in F(\mathbb{R})$ and $\lambda \in \mathbb{R}$. If $\Omega \subset F(\mathbb{R}), \lambda$. $\Omega \subset \Omega, \forall \lambda \in \mathbb{R}$ and $\omega(A) \in \Omega$ is the nearest fuzzy number to a given $A \in F(\mathbb{R})$ (with respect to $D$ ) which preserves $P_{k}, k \in\{1, \ldots, n\}$, that is

$$
P_{k}(\omega(A))=P_{k}(A), \forall k \in\{1, \ldots, n\}
$$

then $\lambda \cdot \omega(A) \in \Omega$ is the nearest fuzzy number to $\lambda \cdot A$ (with respect to $D$ ) which preserves $P_{k}, k \in$ $\{1, \ldots, n\}$, that is

$$
P_{k}(\omega(\lambda \cdot A))=P_{k}(\lambda \cdot A), \forall k \in\{1, \ldots, n\}
$$

Proof. First, we consider the case $\lambda=0$. Since $0 \in$ $\Omega$, it follows that $\omega(0)=0$. This implies $\omega(\lambda \cdot A)=$ $\omega(0)=0=\lambda \cdot \omega(A)$. Therefore, in what follows we may suppose that $\lambda \neq 0$.

We have

$$
D(A, \omega(A)) \leq D(A, M),
$$

for every $M \in \Omega$ such that $P_{k}(A)=P_{k}(M), \forall k \in$ $\{1, \ldots, n\}$ and

$$
P_{k}(A)=P_{k}(\omega(A)), \forall k \in\{1, \ldots, n\} .
$$

Let $A \in F(\mathbb{R})$ and $\lambda \in \mathbb{R}$. Let $M^{\prime} \in \Omega$ such that $P_{k}(\lambda \cdot A)=P_{k}\left(M^{\prime}\right), \forall k \in\{1, \ldots, n\}$. According to (17) and (18) we have $P_{k}(A)=P_{k}\left(\frac{1}{\lambda} \cdot M^{\prime}\right), \forall k \in$ $\{1, \ldots, n\}$. We get

$$
\begin{aligned}
& D(\lambda \cdot A, \lambda \cdot \omega(A))=|\lambda| D(A, \omega(A)) \\
& \leq|\lambda| D\left(A, \frac{1}{\lambda} \cdot M^{\prime}\right)=D\left(\lambda \cdot A, M^{\prime}\right)
\end{aligned}
$$

and

$$
\begin{gathered}
P_{k}(\lambda \cdot A)=\lambda P_{k}(A) \\
=\lambda P_{k}(\omega(A))=P_{k}(\lambda \cdot \omega(A))
\end{gathered}
$$

or

$$
\begin{gathered}
P_{k}(\lambda \cdot A)=|\lambda| P_{k}(A) \\
=|\lambda| P_{k}(\omega(A))=P_{k}(\lambda \cdot \omega(A)),
\end{gathered}
$$

$\forall k \in\{1, \ldots, n\}$, therefore $\lambda \cdot \omega(A) \in \Omega$ is the nearest fuzzy number to $\lambda \cdot A$ (with respect to $D$ ) which preserves the parameters $P_{k}, k \in\{1, \ldots, n\}$. In other words,

$$
\omega(\lambda \cdot A)=\lambda \cdot \omega(A) .
$$

Remark 5 Note that the presumption $\lambda \cdot \Omega \subset$ $\Omega, \forall \lambda \in \mathbb{R}$ in Theorem 4 (as the presumption $z+\Omega=$ $\Omega, \forall z \in \mathbb{R}$ in Theorem 1) is important. Indeed, if $r, s>0, r \neq s$ and

$\Omega=\{A \in F(\mathbb{R}): \exists a, b, \sigma, \beta \in \mathbb{R}, a \leq b, \sigma \geq 0, \beta \geq 0$ such that $A_{L}(\alpha)=a-\sigma(1-\alpha)^{1 / r}$,

$$
\left.A_{U}(\alpha)=b+\beta(1-\alpha)^{1 / s}, \forall \alpha \in[0,1]\right\}
$$

then $\lambda \cdot \Omega \varsubsetneqq \Omega$ for $\lambda<0$. The operator $\omega: F(\mathbb{R}) \rightarrow$ $\Omega$ such that $\omega(A) \in \Omega$ is the nearest fuzzy number to given $A$ with respect to distance d (see (2)) is not scale invariant (see [3], Theorem 12, (iii)) even if every other hypotheses in Theorem 4 are satisfied.
Corollary 6 Let $P_{k}: F(\mathbb{R}) \rightarrow \mathbb{R}, k \in\{1, \ldots, n\}$ defined by

$$
\begin{aligned}
P_{k}(A) & =a_{k} \int_{0}^{1} A_{L}(\alpha) d \alpha+b_{k} \int_{0}^{1} A_{U}(\alpha) d \alpha \\
& +g_{k} \int_{0}^{1} \alpha A_{L}(\alpha) d \alpha+h_{k} \int_{0}^{1} \alpha A_{U}(\alpha) d \alpha
\end{aligned}
$$

where $a_{k}, b_{k}, g_{k}, h_{k} \in \mathbb{R}, \Omega \subset F(\mathbb{R})$ with the property $\lambda \cdot \Omega \subset \Omega, \forall \lambda \in \mathbb{R}$ and $\omega: F(\mathbb{R}) \rightarrow \Omega$ such that $\omega(A)$ is the nearest fuzzy number to $A$ with respect to a scale invariant distance $D$, which, in addition, preserves the parameters $P_{k}, k \in\{1, \ldots, n\}$. If

$$
\begin{gathered}
a_{k}=b_{k}, \\
g_{k}=h_{k}
\end{gathered}
$$

or

$$
\begin{aligned}
b_{k} & =-a_{k}, \\
h_{k} & =-g_{k},
\end{aligned}
$$

for every $k \in\{1, \ldots, n\}$, then

$$
\omega(\lambda \cdot A)=\lambda \cdot \omega(A),
$$

for every $\lambda \in \mathbb{R}$.

Proof. In this particular case (17) is equivalent with (19)-(20) and (18) is equivalent with (21)-(22).

Corollary 7 Let $P_{k}: F(\mathbb{R}) \rightarrow \operatorname{Int}(\mathbb{R}), k \in$ $\{1, \ldots, n\}$ defined by

$$
P_{k}(A)=\left[P_{k}^{\prime}(A), P_{k}^{\prime \prime}(A)\right],
$$

where

$$
\begin{aligned}
P_{k}^{\prime}(A) & =a_{k}^{\prime} \int_{0}^{1} A_{L}(\alpha) d \alpha+b_{k}^{\prime} \int_{0}^{1} A_{U}(\alpha) d \alpha \\
& +g_{k}^{\prime} \int_{0}^{1} \alpha A_{L}(\alpha) d \alpha+h_{k}^{\prime} \int_{0}^{1} \alpha A_{U}(\alpha) d \alpha
\end{aligned}
$$

$$
\begin{aligned}
P_{k}^{\prime \prime}(A) & =a_{k}^{\prime \prime} \int_{0}^{1} A_{L}(\alpha) d \alpha+b_{k}^{\prime \prime} \int_{0}^{1} A_{U}(\alpha) d \alpha \\
& +g_{k}^{\prime \prime} \int_{0}^{1} \alpha A_{L}(\alpha) d \alpha+h_{k}^{\prime \prime} \int_{0}^{1} \alpha A_{U}(\alpha) d \alpha
\end{aligned}
$$

$a_{k}^{\prime}, b_{k}^{\prime}, g_{k}^{\prime}, h_{k}^{\prime}, a_{k}^{\prime \prime}, b_{k}^{\prime \prime}, g_{k}^{\prime \prime}, h_{k}^{\prime \prime} \in \mathbb{R}, \Omega \subset F(\mathbb{R})$ with the property $\lambda \cdot \Omega \subset \Omega, \forall \lambda \in \mathbb{R}$ and $\omega: F(\mathbb{R}) \rightarrow \Omega$ such that $\omega(A)$ is the nearest fuzzy number to $A$ with respect to a scale invariant distance $D$ which, in addition, preserves $P_{k}, k \in\{1, \ldots, n\}$. If

$$
\begin{aligned}
a_{k}^{\prime} & =b_{k}^{\prime \prime}, \\
g_{k}^{\prime} & =h_{k}^{\prime \prime}, \\
a_{k}^{\prime \prime} & =b_{k}^{\prime}, \\
g_{k}^{\prime \prime} & =h_{k}^{\prime}
\end{aligned}
$$


or

$$
\begin{aligned}
a_{k}^{\prime} & =-b_{k}^{\prime}, \\
g_{k}^{\prime} & =-h_{k}^{\prime}, \\
a_{k}^{\prime \prime} & =-b_{k}^{\prime \prime}, \\
g_{k}^{\prime \prime} & =-h_{k}^{\prime \prime}
\end{aligned}
$$

for every $k \in\{1, \ldots, n\}$, then

$$
\omega(\lambda \cdot A)=\lambda \cdot \omega(A),
$$

for every $\lambda \in \mathbb{R}$.

Proof. In this particular case (17) is equivalent with (23)-(26) and (18) is equivalent with (27)-(30).

\section{Applications to crisp, interval, triangular, trapezoidal and parametric approximations of fuzzy numbers}

With respect to the hypothesis in Theorems 1 and 4 , let us remark that the distances $d_{\lambda}, d, \delta_{p, q}, \delta_{\infty, q}, d_{\infty}, d_{p}, \widetilde{D}_{f, \varphi}, D_{\psi, \theta}^{*}$ on $F(\mathbb{R})$, introduced by (1)-(8), are invariant to translations and $d, d_{\infty}, d_{p}, D_{\psi, \theta}^{*}$ are scale invariant. If $\lambda_{L}(\alpha)=$ $\lambda_{U}(\alpha)$, for every $\alpha \in[0,1]$, then $d_{\lambda}$ is scale invariant too.

Let us consider the following subsets of $F(\mathbb{R})$ :

$$
\begin{gathered}
\mathbb{R}^{c}=\{A \in F(\mathbb{R}): \exists c \in \mathbb{R} \text { such that } \\
\left.A_{L}(\alpha)=A_{U}(\alpha)=c, \forall \alpha \in[0,1]\right\}, \\
\mathbb{I}=\left\{A \in F(\mathbb{R}): \exists c_{L}, c_{U} \in \mathbb{R}\right. \text { such that } \\
\left.A_{L}(\alpha)=c_{L}, A_{U}(\alpha)=c_{U}, \forall \alpha \in[0,1]\right\}, \\
\triangle=\left\{A \in F(\mathbb{R}): \exists t_{1}, t_{2}, t_{3} \in \mathbb{R}, t_{1} \leq t_{2} \leq t_{3}\right. \\
\text { such that } A_{L}(\alpha)=t_{1}+\alpha\left(t_{2}-t_{1}\right), \\
\left.A_{U}(\alpha)=t_{3}+\alpha\left(t_{2}-t_{3}\right), \forall \alpha \in[0,1]\right\}, \\
\triangle^{s}=\left\{A \in F(\mathbb{R}): \exists t_{1}, t_{2}, t_{3} \in \mathbb{R}, t_{1} \leq t_{2} \leq t_{3},\right. \\
t_{3}-t_{2}=t_{2}-t_{1} \text { such that } A_{L}(\alpha)=t_{1} \\
\left.+\alpha\left(t_{2}-t_{1}\right), A_{U}(\alpha)=t_{3}+\alpha\left(t_{2}-t_{3}\right), \forall \alpha \in[0,1]\right\}, \\
\mathbb{T}=\left\{A \in F(\mathbb{R}): \exists t_{1}, t_{2}, t_{3}, t_{4} \in \mathbb{R}, t_{1} \leq t_{2} \leq\right. \\
t_{3} \leq t_{4} \text { such that } A_{L}(\alpha)=t_{1}+\alpha\left(t_{2}-t_{1}\right), \\
\left.A_{U}(\alpha)=t_{4}+\alpha\left(t_{3}-t_{4}\right), \forall \alpha \in[0,1]\right\}
\end{gathered}
$$

and

$$
\begin{aligned}
& \mathbb{P}_{s}=\{A \in F(\mathbb{R}): \exists a, b, \sigma, \beta \in \mathbb{R}, a \leq b, \sigma \geq 0, \beta \geq 0 \\
& \text { such that } A_{L}(\alpha)=a-\sigma(1-\alpha)^{1 / s}, \\
& \left.A_{U}(\alpha)=b+\beta(1-\alpha)^{1 / s}, \forall \alpha \in[0,1]\right\}
\end{aligned}
$$

where $s>0$ is fixed. In fact, $\mathbb{R}^{c}$ is the set of real numbers, $\mathbb{I}$ is the set of real intervals, $\Delta$ is the set of triangular fuzzy numbers, $\triangle^{s}$ is the set of symmetric triangular fuzzy numbers, $\mathbb{T}$ is the set of trapezoidal fuzzy numbers and $\mathbb{P}_{s}$ is the set of parametric $(s, s)$-fuzzy numbers (see [15]). If $\Omega \in\left\{\mathbb{R}^{c}, \mathbb{I}, \triangle, \triangle^{s}, \mathbb{T}, \mathbb{P}_{s}\right\}$ then $z+\Omega=\Omega$, for every $z \in \mathbb{R}$ and $\lambda \cdot \Omega \subset \Omega$, for every $\lambda \in \mathbb{R}$.

The following results are immediate consequences of Theorems 1 and 4.

Corollary $8(i)$ The operator $O_{\mathbb{R}^{c}}: F(\mathbb{R}) \rightarrow \mathbb{R}^{c}$, where $O_{\mathbb{R}^{c}}(A)$ is the nearest crisp value to $A$ with respect to distance $d$ in (2), is scale and translation invariant.

(ii) The operator $O_{\mathbb{I}}: F(\mathbb{R}) \rightarrow \mathbb{I}$, where $O_{\mathbb{I}}(A)$ is the nearest interval to $A$ with respect to distance $d$, is scale and translation invariant.

(iii) The operator $O_{\triangle}: F(\mathbb{R}) \rightarrow \triangle$, where $O_{\triangle}(A)$ is the nearest triangular fuzzy number to $A$ with respect to distance $d$, is scale and translation invariant.

(iv) The operator $O_{\mathbb{T}}: F(\mathbb{R}) \rightarrow \mathbb{T}$, where $O_{\mathbb{T}}(A)$ is the nearest trapezoidal fuzzy number to $A$ with respect to distance $d$, is scale and translation invariant.

$(v)$ The operator $O_{\mathbb{P}_{s}}: F(\mathbb{R}) \rightarrow \mathbb{P}_{s}$, where $O_{\mathbb{P}_{s}}(A)$ is the nearest $(s, s)$-fuzzy number to $A$ with respect to distance $d$, is scale and translation invariant.

(vi) The operator $O_{\triangle^{s}}: F(\mathbb{R}) \rightarrow \triangle^{s}$, where $O_{\triangle s}(A)$ is the nearest symmetric triangular fuzzy number to $A$ with respect to distance $d$, is scale and translation invariant.

In fact, $O_{\mathbb{R}^{c}}(A)=E V(A)$ and $O_{\mathbb{I}}(A)=E I(A)$, for every $A \in F(\mathbb{R})$, therefore the scale and translation invariance in $(i)$ and $(i i)$ are consequences of the properties of the expected value and expected interval, respectively. The scale and translation invariance of $O_{\triangle}, O_{\mathbb{T}}, O_{\mathbb{P}_{s}}$ are already proved in [17] and [3]. The operator $O_{\triangle^{s}}$ was determined in [14], but its properties have not been studied yet.

Corollary $9(i)$ The operator $O_{\mathbb{R}^{c}}^{w}: F(\mathbb{R}) \rightarrow \mathbb{R}^{c}$, where $O_{\mathbb{R}^{c}}^{w}(A)$ is the nearest crisp value to $A$ with respect to distance $d_{\lambda}$ in (1), is translation invariant. If $\lambda_{L}(\alpha)=\lambda_{U}(\alpha)$, for every $\alpha \in[0,1]$, then it is scale invariant too.

(ii) The operator $O_{\mathbb{I}}^{w}: F(\mathbb{R}) \rightarrow \mathbb{I}$, where $O_{\mathbb{I}}^{w}(A)$ is the nearest interval to $A$ with respect to distance $d_{\lambda}$, is translation invariant. If $\lambda_{L}(\alpha)=\lambda_{U}(\alpha)$, for every $\alpha \in[0,1]$, then it is scale invariant too.

(iii) The operator $O_{\triangle}^{w}: F(\mathbb{R}) \rightarrow \triangle$, where $O_{\triangle}^{w}(A)$ is the nearest triangular fuzzy number to $A$ with respect to distance $d_{\lambda}$, is translation invariant. If $\lambda_{L}(\alpha)=\lambda_{U}(\alpha)$, for every $\alpha \in[0,1]$, then it is scale invariant too.

(iv) The operator $O_{\mathbb{T}}^{w}: F(\mathbb{R}) \rightarrow \mathbb{T}$, where $O_{\mathbb{T}}^{w}(A)$ is the nearest trapezoidal fuzzy number to $A$ with respect to distance $d_{\lambda}$, is translation invariant. If $\lambda_{L}(\alpha)=\lambda_{U}(\alpha)$, for every $\alpha \in[0,1]$, then it is scale invariant too. 
(v) The operator $O_{\mathbb{P}_{s}}^{w}: F(\mathbb{R}) \rightarrow \mathbb{P}_{s}$, where $O_{\mathbb{P}_{s}}^{w}(A)$ is the nearest $(s, s)$-fuzzy number to $A$ with respect to distance $d_{\lambda}$, is translation invariant. If $\lambda_{L}(\alpha)=\lambda_{U}(\alpha)$, for every $\alpha \in[0,1]$, then it is scale invariant too.

The translation and scale invariance of $O_{\triangle}^{w}$ and $O_{\mathbb{T}}^{w}$ are already studied in [18], and the properties of $O_{\mathbb{P}_{s}}^{w}$ in [19]. Even if the nearest crisp value and the nearest interval to a given $A \in F(\mathbb{R})$, with respect to $d_{\lambda}$, are not determined yet, their existence and uniqueness are obvious and the results in $(i)$ and (ii) are valid for these approximation operators too.

The existence and uniqueness of the nearest crisp value, interval, triangular fuzzy number, trapezoidal fuzzy numbers or parametric $(s, s)$-fuzzy number with respect to distances $\delta_{p, q}, \delta_{\infty, q}, d_{\infty}, d_{p}, \widetilde{D}_{f, \varphi}, D_{\psi, \theta}^{*}$ on $F(\mathbb{R})$, introduced by (3)-(8), could lead towards analogous results with Corollaries 8 and 9 .

In the sequel we give some results related with trapezoidal approximations of fuzzy numbers.

With the notations in Theorems 1 and 4, Corollaries 3 and 7 , we consider $n=1$ and

$$
\begin{gathered}
P_{1}(A)=\left[P_{1}^{\prime}(A), P_{1}^{\prime \prime}(A)\right] \\
=E I(A)=\left[\int_{0}^{1} A_{L}(\alpha) d \alpha, \int_{0}^{1} A_{U}(\alpha) d \alpha\right],
\end{gathered}
$$

that is

$$
\begin{aligned}
& a_{1}^{\prime}=1, b_{1}^{\prime}=g_{1}^{\prime}=h_{1}^{\prime}=0, \\
& a_{1}^{\prime \prime}=g_{1}^{\prime \prime}=h_{1}^{\prime \prime}=0, b_{1}^{\prime \prime}=1 .
\end{aligned}
$$

The following result, already proved in [2] is immediate.

Corollary 10 The operator $O_{E I}: F(\mathbb{R}) \rightarrow \mathbb{T}$, where $O_{E I}(A)$ is the nearest trapezoidal fuzzy number to $A$ (with respect to distance $d$ in (2)), which preserves the expected interval, is scale and translation invariant.

With the notations in Theorems 1 and 4, Corollaries 2 and 6 , we consider $n=2$ and

$$
\begin{gathered}
P_{1}(A)=\operatorname{Amb}(A) \\
=\int_{0}^{1} \alpha A_{U}(\alpha) d \alpha-\int_{0}^{1} \alpha A_{L}(\alpha) d \alpha \\
P_{2}(A)=\operatorname{Val}(A) \\
=\int_{0}^{1} \alpha A_{U}(\alpha) d \alpha+\int_{0}^{1} \alpha A_{L}(\alpha) d \alpha
\end{gathered}
$$

that is

$$
\begin{aligned}
& a_{1}=b_{1}=0, g_{1}=-1, h_{1}=1 \\
& a_{2}=b_{2}=0, g_{2}=h_{2}=1
\end{aligned}
$$

The following result, already proved in [4] is immediate.
Corollary 11 The operator $O_{A m b, V a l}: F(\mathbb{R}) \rightarrow$ $\mathbb{T}$, where $O_{A m b, V a l}(A)$ is the nearest trapezoidal fuzzy number to $A$ (with respect to distance $d$ in (2)), which preserves the ambiguity and value, is scale and translation invariant.

We consider the operator $O_{\text {core }}: F(\mathbb{R}) \rightarrow \mathbb{T}$, where $O_{\text {core }}(A)$ is the nearest trapezoidal fuzzy number to $A$ (with respect to distance $d$ in (2)), which preserves the core, that is

$$
\operatorname{core}\left(O_{\text {core }}(A)\right)=\operatorname{core}(A)=\left[A_{L}(1), A_{U}(1)\right] \text {. }
$$

If $n=1$ and $P_{1}(A)=\left[A_{L}(1), A_{U}(1)\right]$ then

$$
\begin{aligned}
P_{1}(A+z) & =\left[A_{L}(1)+z, A_{U}(1)+z\right] \\
& =P_{1}(A)+z,
\end{aligned}
$$

for every $z \in \mathbb{R}$,

$$
P_{1}(\lambda \cdot A)=\left[\lambda A_{L}(1), \lambda A_{U}(1)\right]=\lambda P_{1}(A),
$$

for every $\lambda \in \mathbb{R}, \lambda \geq 0$ and

$$
P_{1}(\lambda \cdot A)=\left[\lambda A_{U}(1), \lambda A_{L}(1)\right]=\lambda P_{1}(A),
$$

for every $\lambda \in \mathbb{R}, \lambda<0$, therefore from Theorems 1 and 4 we obtain the following result, already proved in [1].

Corollary 12 The operator $O_{\text {core }}: F(\mathbb{R}) \rightarrow \mathbb{T}$ is scale and translation invariant.

An important benefit of the results in Theorems 1 and 4 is the possibility to deduce the properties of scale and translation invariance even if the approximations are actually unknown. For example, the calculus of the nearest trapezoidal fuzzy number to a fuzzy number, which preserves the ambiguity and the nearest trapezoidal fuzzy number to a fuzzy number, which preserves the value, both with respect to distance $d$ in (2) were not performed yet. With the notations in Theorems 1 and 4, Corollaries 2 and 6 , we consider $n=1$ and

$$
\begin{gathered}
P_{1}(A)=\operatorname{Amb}(A) \\
=\int_{0}^{1} \alpha A_{U}(\alpha) d \alpha-\int_{0}^{1} \alpha A_{L}(\alpha) d \alpha,
\end{gathered}
$$

then

$$
\begin{gathered}
P_{1}(A)=\operatorname{Val}(A) \\
=\int_{0}^{1} \alpha A_{U}(\alpha) d \alpha+\int_{0}^{1} \alpha A_{L}(\alpha) d \alpha
\end{gathered}
$$

that is

$$
a_{1}=b_{1}=0, g_{1}=-1, h_{1}=1,
$$

then

$$
a_{1}=b_{1}=0, g_{1}=h_{1}=1,
$$

respectively. We obtain (Corollaries 2 and 6 are used here)

Corollary 13 The operators $O_{A m b}: F(\mathbb{R}) \rightarrow \mathbb{T}$ and $O_{V a l}: F(\mathbb{R}) \rightarrow \mathbb{T}$, where $O_{A m b}(A)$ and $O_{V a l}(A)$ are the nearest trapezoidal fuzzy numbers to $A$ (with respect to distance $d$ in (2)), which preserves the ambiguity and value, respectively, are scale and translation invariant. 


\section{Conclusion}

In the last few years many researchers focused on the approximation of fuzzy numbers. Between the most important requirements that an approximation operator should satisfy are: the invariance to translations, the scale invariance, additivity or continuity. One would expect that an approximation operator would posses as many of this properties as possible. In the present contribution we found sufficient conditions for approximation operators over the space of fuzzy numbers (with respect to wellknown distances) to be invariant to translations and scale invariant, respectively. An immediate consequence of these results is that most of the approximation operator proposed so far in the literature satisfy these requirements. But, according to Theorems 1 and 4, the class of approximation operators which posses this two properties is even larger. This could be an useful tool in finding new approximation operators so that most of the basic requirements are fulfilled. The next goal is to characterize the general class of the additive approximations and/or continuous approximations of fuzzy numbers.

\section{Acknowledgements}

The contribution of the second author was possible with the financial support of the Sectorial Operational Programme for Human Resources Development 2007-2013, co-financed by the European Social Fund, under the project number POSDRU/107/1.5/S/76841 with the title "Modern Doctoral Studies: Internationalization and Interdisciplinarity."

\section{References}

[1] S. Abbasbandy and T. Hajjiri, Weighted trapezoidal approximation-preserving core of a fuzzy number, Computers and Mathematics with Applications, 59:3066-3077, 2010.

[2] A. Ban, Approximation of fuzzy numbers by trapezoidal fuzzy numbers preserving the expected interval, Fuzzy Sets and Systems, 159:1327-1344, 2008.

[3] A. Ban, On the nearest parametric approximation of a fuzzy number-Revisited, Fuzzy Sets and Systems, 160:3027-3047, 2009.

[4] A. Ban, A. Brândaş, L. Coroianu, C. Negruţiu and O. Nica, Approximations of fuzzy numbers by trapezoidal fuzzy numbers preserving the ambiguity and value, Computers and Mathematics with Applications, 61:1379-1401, 2011.

[5] C. Bertoluzza, N. Corral, A. Salas, On a new class of distances between fuzzy numbers, Mathware and Soft Computing, 2:71-84, 1995.

[6] M. Delgado, M.A. Vila and W. Voxman, On a canonical representation of a fuzzy number, Fuzzy Sets and Systems, 93:125-135, 1998.
[7] P. Diamond and P. Kloeden, Metric spaces of fuzzy sets, World Scientific, London, 1994.

[8] D. Dubois and H. Prade, The mean value of a fuzzy number, Fuzzy Sets and Systems, 24:279300, 1987.

[9] P. Grzegorzewski, Metrics and orders in space of fuzzy numbers, Fuzzy Sets and Systems, 97:83-94, 1998.

[10] P. Grzegorzewski and E. Mrówka, Trapezoidal approximations of fuzzy numbers, Fuzzy Sets and Systems, 153:115-135, 2005.

[11] P. Grzegorzewski, Intuitionistic fuzzy numbers - Principles, metrics and ranking, In: Soft Computing. Foundations and Theoretical Aspects, Atanassov, K. T., Hryniewicz, O., Kacprzyk, J. (Eds.), Exit, Warszawa, 2004, pp. 235-249.

[12] S. Heilpern, The expected value of a fuzzy number, Fuzzy Sets and Systems, 47:81-86, 1992.

[13] W.V. Leekwijck and E.E. Kerre, Defuzzification: criteria and classification, Fuzzy Sets and Systems, 108:159-178, 1999.

[14] M. Ma, A. Kandel and M. Friedman, A new approach for defuzzification, Fuzzy Sets and Systems, 111:351-356, 2000.

[15] E.N. Nasibov and S. Peker, On the nearest parametric approximation of a fuzzy number, Fuzzy Sets and Systems, 159:1365-1375, 2008.

[16] W. Trutschnig, G. González-Rodríguez, A. Colubi, M. A. Gil, A new family of metrics for compact, convex (fuzzy) sets based on a generalized concept of mid and spread, Information Sciences, 179:3964-3972, 2009.

[17] C.-T. Yeh, On improving trapezoidal and triangular approximations of fuzzy numbers, International Journal of Approximate Reasoning, 48:297-313, 2008.

[18] C.-T. Yeh, Weighted trapezoidal and triangular approximations of fuzzy numbers, Fuzzy Sets and Systems, 160:3059-3079, 2009.

[19] C.-T. Yeh, Weighted semi-trapezoidal approximations of fuzzy numbers, Fuzzy Sets and Systems, 165:61-80, 2011. 